\title{
Oxide Wizard: An EELS Application to Characterize the White Lines of Transition Metal Edges
}

\author{
Lluís Yedra, ${ }^{1,2, *}$ Elena Xuriguera, ${ }^{3}$ Marta Estrader ${ }^{4}$ Alberto López-Ortega, ${ }^{5}$ Maria D. Baró, ${ }^{6}$ \\ Josep Nogués, ${ }^{7,8}$ Manuel Roldan, ${ }^{9,10}$ Maria Varela, ${ }^{9,10}$ Sònia Estradé, ${ }^{1,2}$ and Francesca Peiró ${ }^{1}$ \\ ${ }^{1}$ Laboratory of Electron Nanoscopies (LENS)-MIND/IN2UB, Dept. d'Electrònica, Universitat de Barcelona, C/Martí i Franquès \\ 1, E-08028 Barcelona, Spain \\ ${ }^{2}$ CCiT, Scientific and Technical Centers, Universitat de Barcelona, C/Lluís Solé i Sabaris 1, E-08028 Barcelona, Spain \\ ${ }^{3}$ Ciència de Materials i Enginyeria Metal-lúrgica, Dept. d'Enginyeria Química, Universitat de Barcelona, E-08028 \\ Barcelona, Spain \\ ${ }^{4}$ Departament de Química Inorgànica, Universitat de Barcelona, Diagonal 645, E-08028, Barcelona, Spain \\ ${ }^{5}$ INSTM and Dipartimento di Chimica "U. Schiff", Università degli Studi di Firenze, Via della Lastruccia 3, Sesto Fiorentino, \\ I-50019 Firenze, Italy \\ ${ }^{6}$ Departament de Física, Universitat Autònoma de Barcelona, E-08193 Bellaterra (Barcelona), Spain \\ ${ }^{7}$ ICN2-Institut Catala de Nanociencia i Nanotecnologia, Campus UAB, E-08193 Bellaterra (Barcelona), Spain \\ ${ }^{8}$ Institució Catalana de Recerca i Estudis Avançats (ICREA), Barcelona, Spain \\ ${ }^{9}$ Oak Ridge National Laboratory, Materials Science \& Technology Division, Oak Ridge, TN 37831, USA \\ ${ }^{10}$ Departamento de Física Aplicada III \& Instituto Pluridisciplinar, Universidad Complutense de Madrid, \\ E-28040 Madrid, Spain
}

\begin{abstract}
Physicochemical properties of transition metal oxides are directly determined by the oxidation state of the metallic cations. To address the increasing need to accurately evaluate the oxidation states of transition metal oxide systems at the nanoscale, here we present "Oxide Wizard." This script for Digital Micrograph characterizes the energy-loss near-edge structure and the position of the transition metal edges in the electron energy-loss spectrum. These characteristics of the edges can be linked to the oxidation states of transition metals with high spatial resolution. The power of the script is demonstrated by mapping manganese oxidation states in $\mathrm{Fe}_{3} \mathrm{O}_{4} /$ $\mathrm{Mn}_{3} \mathrm{O}_{4}$ core/shell nanoparticles with sub-nanometer resolution in real space.
\end{abstract}

Key words: EELS, spectrum image, transition metal oxides, oxidation state, Digital Micrograph script, manganese oxide

\section{INTRODUCTION}

Transition metal (TM) oxides have maintained the interest of researchers for decades owing to their appealing physiochemical properties, which have resulted in many different applications in a broad variety of fields. Interestingly, many of these properties stem directly from their numerous possible oxidation states. Manganite perovskites, for example, have attracted much attention for their colossal magnetoresistance (CMR) properties, which enable applications such as magnetoresistive tunnel junctions (Sun et al., 1997). Actually, CMR is closely related to the electronic inhomogeneity and double exchange ensuing from the $\mathrm{Mn}^{+3} / \mathrm{Mn}^{+4}$ mixed valence present in some doped manganites. Other fields where the TM valence is of great importance include mineralogy (van Aken et al., 1998; Cave et al., 2006; Loomer et al., 2007; Zhang et al., 2010), catalysis (Suchorski et al., 2005), electrochemistry (Graetz et al., 2004), and twodimensional (2D) superconducting materials (Logvenov et al., 2009), among many others.

Oxidation state can be defined as the number of electrons a given atom contributes to the bond, i.e., the

Received September 29, 2013; accepted February 12, 2014

${ }^{*}$ Corresponding author. llyedra@el.ub.edu hypothetical charge the atom would have in a purely ionic picture (McNaught \& Wilkinson, 1997), and it is directly linked to occupation of the $3 d$ energy bands near the Fermi level of the material. However, the formal oxidation state differs from the actual charge on the TM atom (Luo et al., 2007), as the bonds have a marked covalent character.

Importantly, for accurate characterization of complex TM oxides and to properly understand their functional properties, measuring the oxidation state, i.e., the $3 d$ band occupation, is of vital importance. Electron energy-loss spectroscopy (EELS) has been routinely used in order to obtain information on this electronic property, and there are a vast number of publications about the link between the oxidation state and the energy-loss near-edge structure (ELNES) of the $\mathrm{L}_{2,3}$ edges ( $2 p \rightarrow 3 d$ - like transitions) (Rask et al., 1987; Paterson \& Krivanek, 1990; Colliex et al., 1991; Garvie \& Craven, 1994; Botton et al., 1995; Garvie \& Buseck, 1998; Gloter et al., 2001; Turquat et al., 2001; Wang et al., 2001; Daulton et al., 2002; Cave et al., 2006; Kourkoutis et al., 2006; Riedl et al., 2006, 2007, 2008; Schmid \& Mader, 2006; Loomer et al., 2007; Meneses et al., 2007; Arevalo-Lopez et al., 2009; Varela et al., 2009). Many methods have been reported to relate the $\mathrm{L}_{2,3}$ ELNES and the oxidation state, mainly through the measurements of $\mathrm{L}_{2,3}$ intensity ratios. However, most of these methods are not reliable when the 
signal-to-noise ratio (SNR) is poor, as is typically the case in 2D EEL spectrum images (SI) with short acquisition times per pixel. Here, we present Oxide Wizard (OW), a script for Digital Micrograph (DM) that measures and maps several characteristics of the TM edge, which can be related to the oxidation state. The script can be downloaded free from the University of Barcelona digital database (diposit.ub.edu).

\section{BACKGROUND}

Driven by the importance of accurate determination of oxidation state in these materials, several procedures have been accurately compared in the literature in order to correlate features in the ELNES of TM absorption edges with the oxidation state. Here we describe some of those procedures, based on the analysis of the $\mathrm{L}_{2,3}$ edge or the $\mathrm{O} \mathrm{K}$ edge observed in a typical EELS spectrum as in Figure 1:

- White line (WL) ratio: in the case of TMs there are two clear peaks near the $\mathrm{L}_{2,3}$ edge onset. These are the so-called $\mathrm{WL}$, their name arising from the fact that they appear as bright, overexposed stripes in X-rays and still appear as bright stripes in EEL spectra (Leapman et al., 1982). They originate in transitions from the $2 p$ to the $3 d$ bands and their separation corresponds to the spin-orbit splitting from the ground states of the transition $\left(2 p_{3 / 2} \rightarrow 3 d\right)$ and $\left(2 p_{1 / 2} \rightarrow 3 d\right)$. Intuitively, the ratio between the two peaks should also correspond to occupation of the ground states $(2: 1)$ if the final density of states is the same for all electrons. Experimentally, this is not observed in many cases, and changes in the ratio have been successfully related to oxidation states (van Aken et al., 1998; Graetz et al., 2004; Riedl et al., 2006; Schmid \& Mader, 2006; Varela et al., 2009). There are several methods for extracting the WL intensities. Amongst them, the Pearson or step function method (Pearson et al., 1993; Estrade et al., 2012) where a linear model of the continuum is

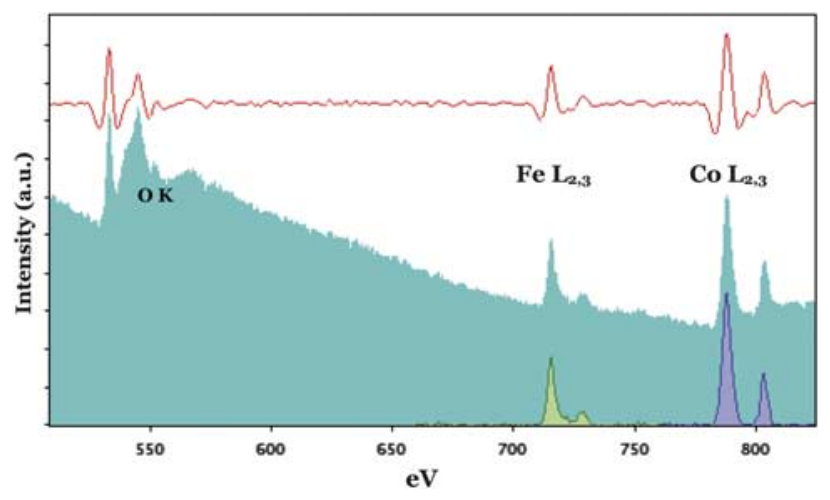

Figure 1. Single spectrum of an iron and cobalt mixed oxide (green filled), including the oxygen $\mathrm{K}$ edge, the $\mathrm{Fe}_{2,3}$ edge, and the Co $\mathrm{L}_{2,3}$ edge. Red shows the second derivative of the spectrum, used for peak detection. Light green shows the Fe white line (WL) after feature localization and continuum subtraction. Blue shows the Co $\mathrm{WL}$ after the same procedure, ready for measurements. subtracted. Measurement of the $\mathrm{L}_{2,3}$ intensity ratios has been one of the most used techniques for oxidation state investigations, but it is very important to keep in mind that characteristics other than oxidation state, such as spin state, coordination geometry, and ligand type, may play an important role (Daulton et al., 2002; Gilbert et al., 2003). Moreover, WL ratio increases with increasing thickness of the sample, but this effect can be removed by deconvolution of the low-loss spectrum (Tan et al., 2012). Methods based on the second derivative (Botton et al., 1995) have also been used.

- TM L edge onset (chemical shift): the onset of edges roughly corresponds to the lowest energy of the final state. The position of the edge will change with changes in binding energy of the core level. In general, the edges move to higher energies for higher oxidation states (Tafto \& Krivanek, 1982; Rask et al., 1987; Daulton et al., 2002; Gilbert et al., 2003; Riedl et al., 2007). This energy shift is usually referred to as chemical shift (Egerton, 1996).

- O K onset: even if the nominal oxidation state of oxygen atoms is -2 , the position of the K edge can be substantially different for different compounds. In manganese compounds, the position of the oxygen $\mathrm{K}$ edge moves monotonically to lower energies for higher oxidation states of the cation (Gilbert et al., 2003; Laffont \& Gibot, 2010; Tan et al., 2012), but this trend is not maintained for all TM oxides.

- $\mathrm{L}_{3}$ full-width at half-maximum (FWHM): two important aspects influence the width of the $\mathrm{L}_{3}$ peak: the oxidation state and the mixture of oxidation states. A slight change in the width of the peak with oxidation state was shown by Riedl et al. (2007) while it had previously been calculated by Sherman (1984) and Kurata \& Colliex (1993). However, the sensitivity to the oxidation state is very low, around $20 \%$ as calculated by Riedl with good SNR. However, the width of the peak can contain information about mixed valences. In the case of $\mathrm{Mn}^{+2.66}$, the observed $\mathrm{L}_{2,3}$ edge results as the overlap of $\mathrm{Mn}^{+2}$ and $\mathrm{Mn}^{+3}$ edges (Tan et al., 2012).

- TM L to O K energy distance: taking into account that the oxygen $\mathrm{K}$ edge onset and that of the TM both shift, the relative distance between the two of them is an interesting feature of the spectrum when no absolute energy positions can be determined. This quantity is related to the energy difference between the $1 s$ level of the oxygen and the $2 p_{3 / 2}$ level of the TM, if we assume the same final state. While it may be equivalent to the TM edge onset if the $\mathrm{O} 1 s$ state remains the same (Arevalo-Lopez et al., 2009), this quantity is generally different because of the nonmonotonicity between the oxidation state and the $\mathrm{O} \mathrm{K}$ edge position and the fact that the final states for the $1 s$ of $\mathrm{O}$ and the $2 p_{3 / 2}$ level of the TM may be different, as they are strongly localized.

- $\mathrm{L}_{2}-\mathrm{L}_{3}$ energy distance: the difference in energy between the $\mathrm{L}_{3}$ and the $\mathrm{L}_{2}$ peaks is a feature rarely used in previous work. Separation of the peaks arises from the spin-orbit splitting from the ground states of the transitions 
$\left(2 p_{3 / 2} \rightarrow 3 d\right)$ and $\left(2 p_{1 / 2} \rightarrow 3 d\right)$. Therefore, any change in the separation is related with the changes in the ground states.

- O K peak-separation method: the energy separation between the $\mathrm{O} \mathrm{K}$ prepeak and the edge main peak is a highly successful and accurate approach to measure oxidation states, which has been tested both experimentally (Varela et al., 2009) and theoretically (Luo et al., 2009). This method, however, is difficult to use in complex heterosystems such as interfaces between two oxides because of beam broadening and cross-talk effects.

All these methods yield consistent results when used on data with good SNR. However, a good SNR is hardly found when working with complex data sets such as 2D SI. Thanks to the recent advances in electron microscopes (Gubbens et al., 2010) the number of spectra available in a single SI is rapidly increasing. Therefore, a procedure to extract reliable information from a great number of spectra at a time is required. In order to address this need, we have developed the OW software, an automated routine for Gatan DM (Gatan Inc., Pleasanton, CA, USA). As we will show, our approach offers a method for characterizing the WL of TM in a systematic manner, including all the necessary tools in a single interface.

\section{Materials and Methods}

To demonstrate the power of the script we have studied the Mn oxidation states in $\mathrm{Fe}_{3} \mathrm{O}_{4} / \mathrm{Mn}_{3} \mathrm{O}_{4}$ core/shell nanoparticles. The nanoparticles were grown using the seeded-growth method (Salazar-Alvarez et al., 2011; Lopez-Ortega et al., 2012) where the $\mathrm{Mn}$ oxide layer was grown on $\mathrm{Fe}_{3} \mathrm{O}_{4} 11 \mathrm{~nm}$ seeds by hot injection (Estrader et al., 2013).

Importantly, to calibrate the script, bulk materials with known oxidation states are necessary. In our case, since we are interested in the $\mathrm{Mn}$ oxidation states, a number of different bulk manganese oxides $\left(\mathrm{MnO}, \mathrm{Mn}_{3} \mathrm{O}_{4}, \mathrm{Mn}_{2} \mathrm{O}_{3}\right.$, and $\mathrm{MnO}_{2}$ ) were synthesized by our colleagues in Ciència de Materials i Enginyeria Metal-lúrgica in the Department of Chemical Engineering of the University of Barcelona. The structure of the samples was confirmed by X-ray diffraction. The calibration was carried out in a JEOL JEM 2010F (JEOL Ltd, Akishima, Tokyo, Japan) operated at $200 \mathrm{kV}$ and equipped with a Gatan GIF spectrometer (Gatan).

EEL SI of the nanoparticles studied were acquired in an aberration-corrected Nion UltraSTEM200 operated at $200 \mathrm{kV}$ and equipped with a Gatan Enfina spectrometer (Gatan) at Oak Ridge National Laboratory.

\section{Steps of the Analysis}

The script, written in DM scripting language, is divided in several sequential steps, which are summarized in the flow chart in Figure 2.

The first three steps are optional and allow the user to improve the quality of the data to ease further analysis. The first step consists in deconvolution of the low-loss region of

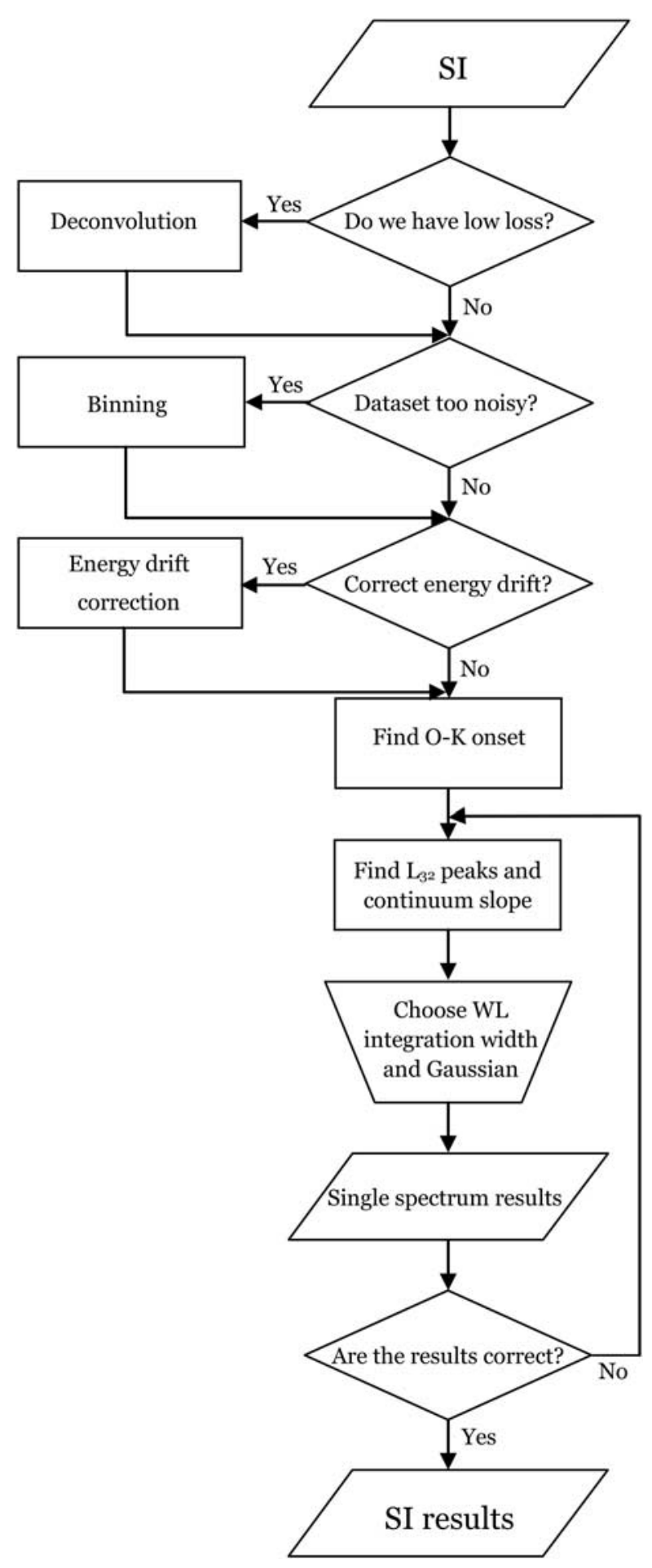

Figure 2. Flow chart of the script, starting with a spectrum image and ending in the results.

the spectrum. Core-loss edges are usually broadened because of the effects of multiple scattering. If the sample is not thin enough, it is likely that the electrons suffering inelastic scattering will also undergo at least a plasmon excitation, broadening the ELNES and, hence, changing the shape of the continuum. If the measurements are carried out in a thin specimen or in an area of the sample with constant thickness 
and only used for comparison purposes, low-loss deconvolution is not necessary. Otherwise, deconvolution of the low-loss region is typically required. This step can be achieved using the Fourier ratio deconvolution already available in the DM EELS package. Binning can be optionally achieved by summing spectra, and it can be helpful when the data set is very noisy or when performing test runs on very large SI.

Next, an energy drift correction procedure can be carried out. This step is based on the presence of peaks other than the TM or oxygen in the spectra. It consists of choosing an edge in one single spectrum with a region of interest (ROI) tool provided by the script. The energy drift is calculated using a cross correlation of that region of the reference spectrum and the rest of the spectra. A new, drift-corrected SI is created. When other peaks are not present, energy drift correction is still recommended. In the subsequent analysis, the user defines the regions where the edges are placed in a single spectrum using a ROI. Energy drift may cause the edges to fall outside the ROI in other spectra. It must be taken into account that the correction of energy drift using the TM edge will affect the absolute peak position determination, but still preserves the peak position relative to the $\mathrm{O} \mathrm{K}$ edge.

Subsequently, the onset of the oxygen $\mathrm{K}$ edge is analyzed. Choosing the first peak of the oxygen with a ROI, the onset is found as a minimum in a modified second derivative (see the red line in Fig. 1), equivalent to the second derivative filter in DM, where maxima in the original function correspond to maxima in the filtered version. The modification inverts the second derivatives in order to locate maxima that correspond to minima in the actual second derivative of the function.

Finally, the ELNES of the TM is characterized by finding the positions of the maxima of the two WL, the onset of the $\mathrm{L}_{3}$, the minimum in between, and the end of the $\mathrm{L}_{2}$. It must be noticed that before this analysis the background needs to be subtracted (by using power-law fits in DM or other models). After placing a ROI on the WL of a single spectrum, the script calculates the second derivative, finds the maximum, and fits a Gaussian curve to it. This maximum corresponds to the first WL. This curve is then used to calculate the position of the other features by cross correlating it with the second derivative of the spectrum. A cross correlation is used since it is easily and rapidly calculated in DM, it is robust against noise, and it enhances actual WL peaks in the second derivative against local maxima and minima.

The cross correlation has a few distinct features: a global maximum placed at zero position and a series of peaks that match the greater features of the second derivative of the spectrum. The minimum before the first WL is taken as the onset of the edge. The global maximum not corresponding to the zero position, found as the maximum of the cross correlation after a few electron volts from the zero, corresponds to the second WL. The two other features are less important for the analysis, but are calculated for further data treatment. The valley between the WL and the end of the ELNES are found as the minima after the first and second $\mathrm{WL}$, respectively. The end of the ELNES is shown to avoid overlapping with the continuum calculation. The five features are plotted on the spectrum to enable visual detection of eventual errors in the localization. Once the features of the ELNES are located, we already have the information about the absolute positions of the lines. However, if we want to integrate the areas under the peaks, it is necessary to remove the contribution of the continuum (Pearson et al., 1993). A few methods have been devised for this purpose, such as modeling it with Hartree-Slater cross-sections. This method calculates the continuum contribution with a neutral atom cross-section. However, a simpler alternative is a linear fitting of the continuum, i.e., the Pearson method, which has been chosen for its simplicity and for having results similar to those of Walsh Dray (Riedl et al., 2006). For this method, the continuum is modeled as a two-step function with the steps centered under the peaks, with a height ratio of 2:1 from first to second, and with a slope calculated by a linear fit of the continuum after the peaks. This function is subtracted from the data before the intensity under the peaks is calculated (see the extracted WL in Fig. 1). The script allows the user to decide the width of the integrating windows and also the continuum modeling region.

The script can present results for a single spectrum or for the whole SI. In both cases the results can be refined by fitting the peaks after the continuum subtraction with two Gaussian curves. The final results provided by the script are the TM edge onset, the WL $\mathrm{L}_{2,3}$ ratio, the energy split between the two WLs, the energy between the $\mathrm{O} K$ and the TM $\mathrm{L}_{2,3}$ edge and the FWHM of the first WL.

\section{Results}

\section{Calibration Test on $\mathrm{Mn}_{x} \mathrm{O}_{y}$ Oxides}

In Figure 3, single spectra for the four different bulk manganese oxides considered $\left(\mathrm{MnO}, \mathrm{Mn}_{3} \mathrm{O}_{4}, \mathrm{Mn}_{2} \mathrm{O}_{3}\right.$, and $\mathrm{MnO}_{2}$ ) are shown. The differences in the $\mathrm{O} \mathrm{K}$ edges and the $\mathrm{Mn} \mathrm{L}_{2,3}$ edges are clear, the $\mathrm{L}_{3} / \mathrm{L}_{2}$ ratio decreases with oxidation state and the $\mathrm{O} \mathrm{K}$ onset position increases. Rather than calibration curves, we preferred the two-parameter

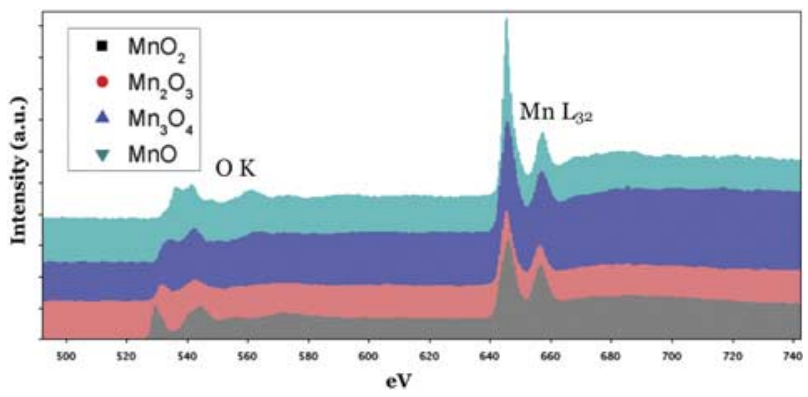

Figure 3. Single spectra of the different manganese oxide reference materials used for data extraction for $\mathrm{Mn}$ formal oxidation ratios ranging from two to four, displaced in the intensity direction. The spectra are aligned at the $\mathrm{Mn} \mathrm{L}_{2,3}$ edge. 
a

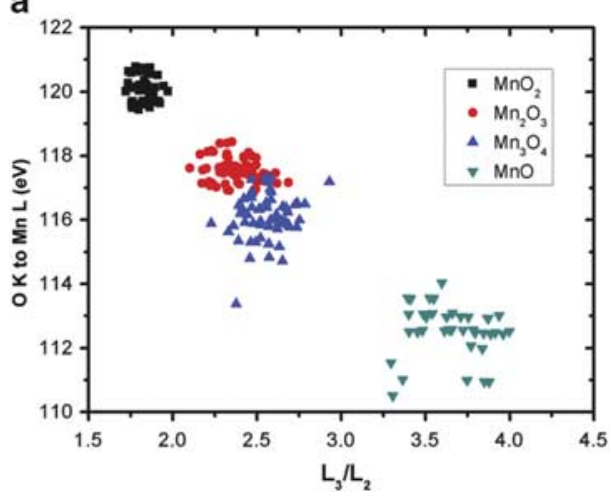

b

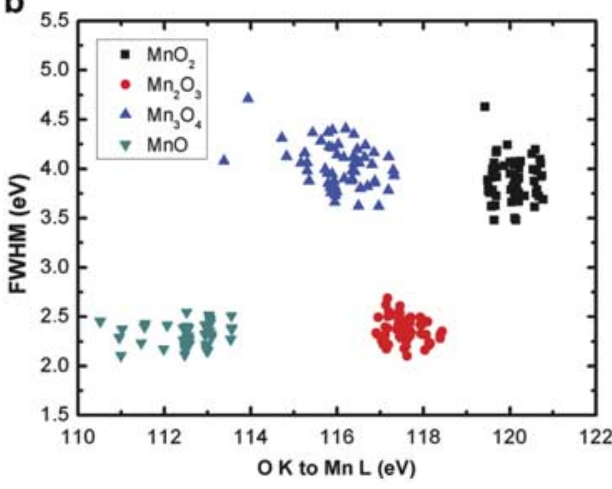

Figure 4. Two-parameter chart combining (a) white line ratios and $\mathrm{O} K$ to $\mathrm{Mn} \mathrm{L}_{3}$ distances and (b) $\mathrm{O} \mathrm{K}$ to $\mathrm{Mn} \mathrm{L}_{3}$ distances and full-width at half-maximum of the $\mathrm{L}_{3}$ peak for $\mathrm{Mn}$ formal oxidation ratios ranging from two to four.

method introduced by Daulton et al. (2002) in chromium and performed by Tan et al. (2012) on manganese and iron oxides. This method presents the results of the two WL features in the same plot. While a single parameter can be misleading, the two-parameter method makes the characterization easier. The results of this procedure are plotted as two-parameter charts in Figure 4. The $\mathrm{Mn} \mathrm{L}_{2,3}$ onset is discarded, as there is no absolute measure of the position. Similarly, the $\mathrm{L}_{3}-\mathrm{L}_{2}$ distance is not used since it did not show relevant changes in the data. The $\mathrm{WL}$ ratio did show the expected changes, and in Figure 4a it is plotted against the $\mathrm{O} K$ to $\mathrm{Mn}_{3}$ distances, which also showed a large change between oxidation states. In the chart, although the different formal oxidation states are separated, the $\mathrm{Mn}_{3} \mathrm{O}_{4}$ (in red) and $\mathrm{Mn}_{2} \mathrm{O}_{3}$ (in blue) show data clouds very close to one another, with some data points overlapping. To avoid ambiguity in the analysis, a third parameter, FWHM of $\mathrm{L}_{3}$ peak, is introduced. As seen in Figure $4 \mathrm{~b}$, in this case all the data clouds, and particularly $\mathrm{Mn}_{3} \mathrm{O}_{4}$ and $\mathrm{Mn}_{2} \mathrm{O}_{3}$ ones, are well separated. Deviation of the $\mathrm{WL}$ characteristics with respect to the measured mean value is below $6 \%$ for the WL ratio, below 9\% for the FWHM, and below $1 \%$ for the $\mathrm{O} \mathrm{K}$ to $\mathrm{Mn}_{3}$ distances.

$\mathrm{Mn}_{2} \mathrm{O}_{3}$ has a single oxidation state, with $\mathrm{Mn}^{3+}$, while $\mathrm{Mn}_{3} \mathrm{O}_{4}$ contains a mixture of $\mathrm{Mn}^{3+}$ and $\mathrm{Mn}^{2+}$ in a 2:1 ratio. The measured edge can be seen as a superposition of both oxidation states. The onset of the edge for $\mathrm{Mn}_{3} \mathrm{O}_{4}$ is therefore close to that of the $\mathrm{Mn}_{2} \mathrm{O}_{3}$ edge, both given by the $\mathrm{Mn}^{3+}$ contribution. However, there was a variation in $\mathrm{O} K$ position, so the distance between $\mathrm{O}$ and $\mathrm{Mn}$ edges showed a change. The WL ratio is also very close for the two compositions. However, the FWHM of the mixed oxide is expected to be larger, since the $\mathrm{L}_{3}$ will be a superposition of two displaced peaks. The experimental results corroborate this point.

\section{Application of the Script to Determine Mn Oxidation State in Core-Shell Magnetic Nanoparticles}

Once parameters for the edges of the diverse manganese oxide reference materials have been established, the routine can be applied to a sample of unknown oxidation state.
The sample consists of core-shell nanoparticles, where the core corresponds to an iron oxide and the shell to a manganese oxide. A high-angle annular dark-field image of a nanoparticle is shown in Figure 5a. In this image, the core and the inhomogeneous shell are difficult to tell apart. If we carry out a multiple linear least squares (MLLS) fitting of the EELS SI for the iron and manganese edges, we can obtain a composite image with compositional contrast, as shown in Figure $5 \mathrm{~b}$. In this figure, the iron oxide core appears as a round particle presented in red, while the manganese oxide grows as discontinuous crystallites around the core (in green).

The SI has been fitted to two single spectra in Figure 6a from the surface (orange) and the inner part (blue) of the $\mathrm{Mn}$ oxide shell. The two spectra had different characteristics, pointing to different oxidation states. The orange spectrum, extracted from the surface region, has a higher WL ratio, a thinner $\mathrm{L}_{3}$, and an onset shifted to lower energies than the blue spectrum extracted from the inner parts of the manganese oxide shell. The result of the fitting is shown in Figure 6b, where we can observe a distribution of manganese oxidation states. However, the MLLS fitting maps only offer qualitative information if we do not have more prior information about the sample. We then applied OW to assess the oxidation state.

Principal component analysis was applied using Hyperspy, before OW to reduce the noise without binning the data. The script has been applied to the whole spectral image, consisting of $100 \times 70$ single spectra as the one shown in Figure 5c, both with and without low-loss deconvolution. The differences in both procedures were negligible, mainly owing to the small thickness of the particle, which, along with the carbon support, summed only 0.3 inelastic mean free paths. The results of the deconvolved spectra are shown in Figure 7. The Mn WL ratio (Fig. 7a) in the particle ranges from 3 to 3.5. The FWHM of the $\mathrm{L}_{3}$ line (Fig. $7 \mathrm{~b}$ ) has values between 4 and $6 \mathrm{eV}$, and the energy separation between the $\mathrm{O}$ K edge and the $\mathrm{Mn}_{2,3}$ (Fig. 7c) goes from 112 to $115 \mathrm{eV}$.

\section{Discussion}

In the manganese oxide shell, two different regions can be seen corresponding to the surface and the inner areas of 

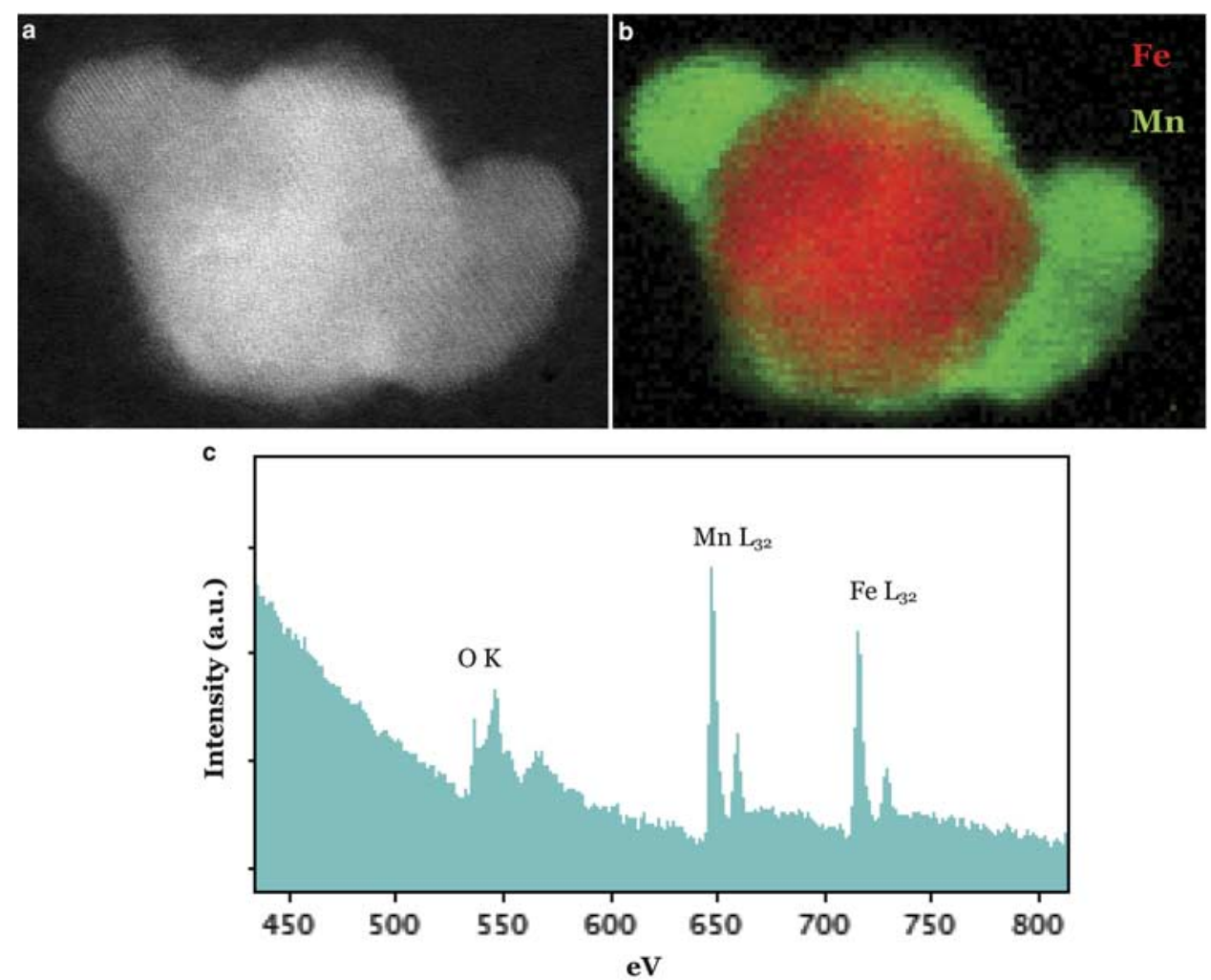

Figure 5. a: High-angle annular dark-field image of the particle and (b) composite image of multiple linear least squares fitting of the spectrum image to the iron and manganese L edges. c: Single spectrum showing the oxygen K, manganese $\mathrm{L}_{2,3}$, and iron $\mathrm{L}_{2,3}$ edges.
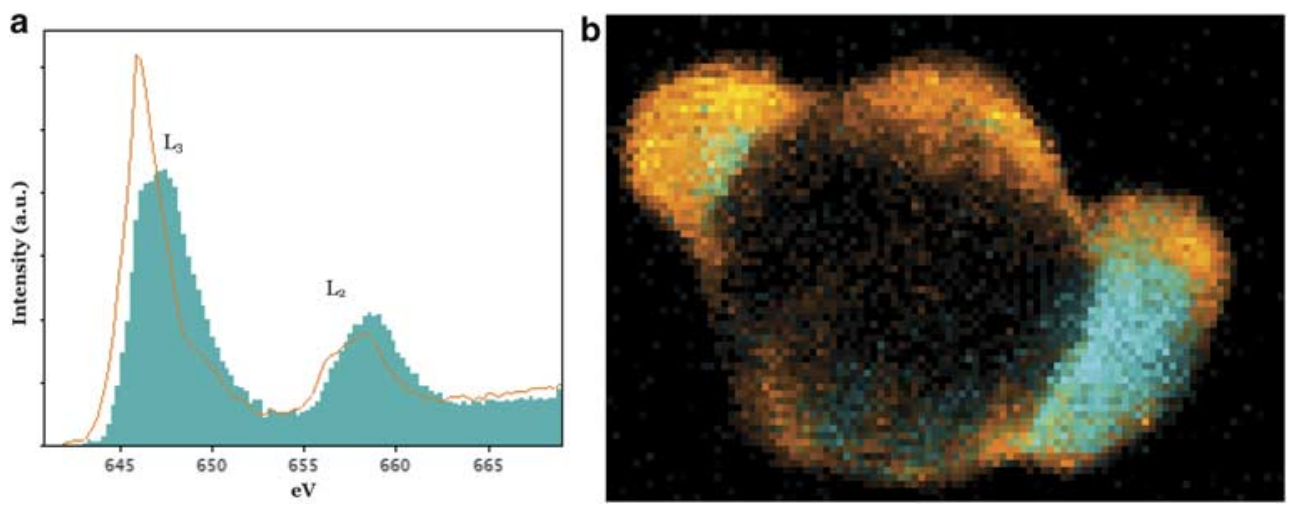

Figure 6. a: $\mathrm{Mn} \mathrm{L}_{2,3}$ edges extracted from the border (in orange) and the inner area (in turquoise) of the manganese oxide shell and (b) composite image of multiple linear least squares fitting of the spectrum image to the two spectra in (a).

the shell. At the surface, the values of both the WL ratio (3.5) and the $\mathrm{O}-\mathrm{Mn}$ distance $(112 \mathrm{eV})$ point to a MnO-like composition according to the two-parameter chart in Figure 4 with an oxidation state of $\mathrm{Mn}^{2+}$. In the inner regions of the shell, displayed in green in Figure 7a and in the range green to yellow in Figure $7 \mathrm{~b}$, the WL ratio is around 3, and the $\mathrm{O}-\mathrm{Mn}$ distance is around $115 \mathrm{eV}$. In Figure 4, however, these values do not correspond to any of the reference oxidation states. The values lie between $\mathrm{Mn}^{2+}$ and $\mathrm{Mn}^{2.66+}$. The spectra for the inner regions of the manganese oxide result from a superposition of the inner and outer areas, as the electron travels through the entire thickness of the sample. Therefore, the actual oxidation state in the inner part must be higher than measured. Using the FWHM measurements, the differences between the surface and the interior of the manganese regions is of $2 \mathrm{eV}$, the same difference found between $\mathrm{Mn}^{2+}$ and $\mathrm{Mn}^{2.66+}$. Therefore, we can assume that the oxidation state of manganese ranges from $\mathrm{Mn}^{2+}$ at the surface to $\mathrm{Mn}^{2.66+}$ in the inside. Interestingly, in our bulk compounds a nominal oxidation state of +2.66 would on average be found in $\mathrm{Mn}_{3} \mathrm{O}_{4}$. Consequently, our results indicate that the shell of the particles may be composed of an 


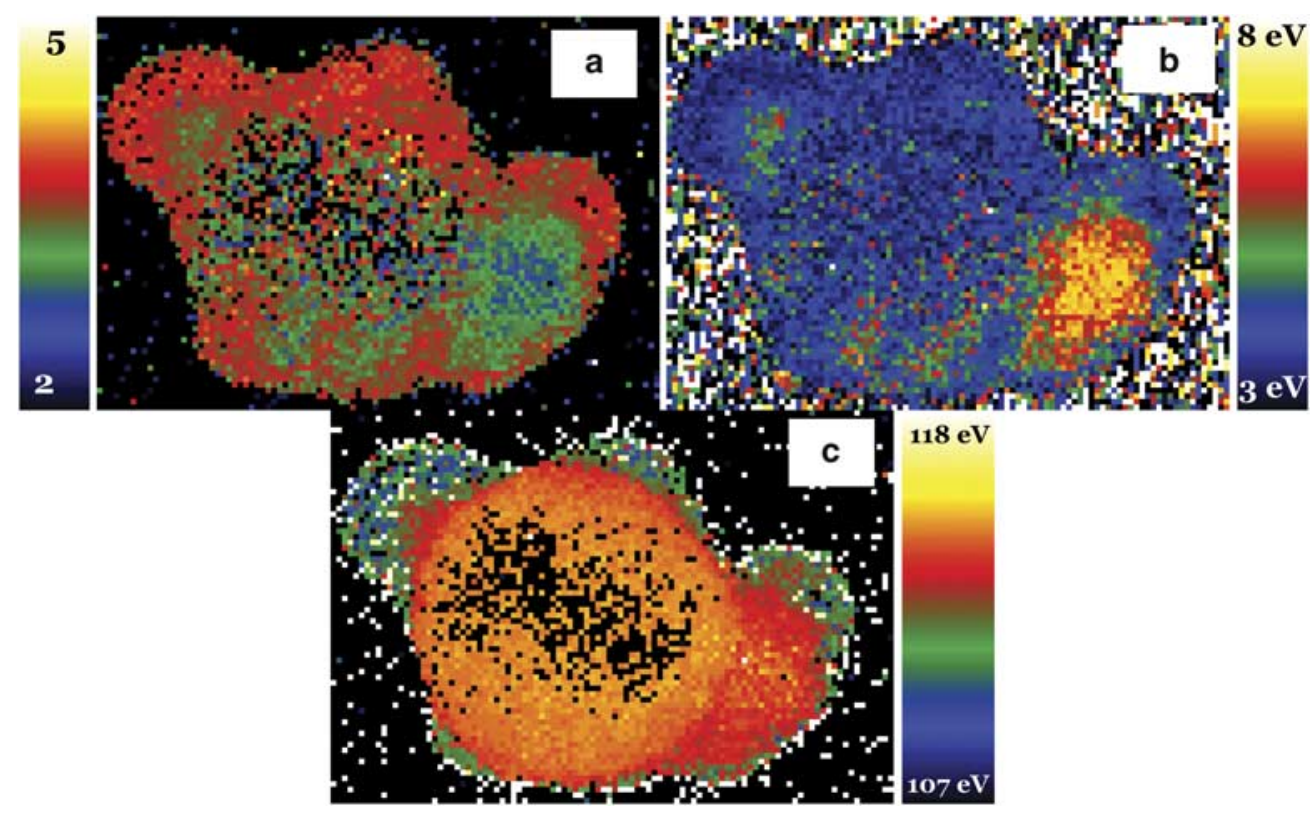

Figure 7. Different outputs of the Oxide Wizard script: (a) White line ratio; (b) $\mathrm{L}_{3}$ full-width at half-maximum after Gaussian fitting; (c) $\mathrm{O} \mathrm{K}$ to $\mathrm{Mn} \mathrm{L}_{3}$ distance.

inhomogeneous $\mathrm{Mn}$ oxide, with a mixture of $\mathrm{MnO}$ at the surface and $\mathrm{Mn}_{3} \mathrm{O}_{4}$ rich areas within the shells.

\section{Conclusions}

A script for DM, OW, has been developed to characterize the WL found in the ELNES of TM, dealing with large spectral images in a systematic way. As an output, the script plots the $\mathrm{WL}$ ratio, the oxygen $\mathrm{K}$ edge onset, the TM edge onset, the difference between both, the FWHM of the TM first WL, and the separation of the WLs. From these parameters, and using the WLs of TM in reference compounds, it is possible to discriminate between compounds with TM having different oxidation states. This procedure has been applied to iron oxide/manganese oxide core/shell nanoparticles with unknown manganese oxidation states. The results show, with sub-nanometer resolution, the existence nanodomains with distinct oxidation states consistent with the presence of $\mathrm{Mn}_{3} \mathrm{O}_{4}$ in the interior of the shell and $\mathrm{MnO}$ rich regions near its surface. Hence, OW is a very effective and reliable script capable of dealing with large data sets enabling real-time processing of data within the DM interface.

\section{ACKNOWLEDGMENTS}

Research at ORNL (M.V.) was sponsored by the Materials Sciences and Engineering Division of the US Department of Energy (DOE). Research at UCM sponsored by the ERC starting Investigator Award, grant \#239739 STEMOX. The authors acknowledge the financial support of the 2009SGR-1292 and 2009 SGR 00035 projects of the Generalitat de Catalunya and the MAT2010-20616-C02, MAT2010-16407, and 2009CSD-00013 projects of the Spanish Ministry of
Science and Innovation (MICINN). D.R.G. Mitchell second derivative and D.R.G. Mitchell and W. Bertram Gaussian fit scripts were used. M.E. acknowledges the Spanish Ministry of Science and Innovation through the Juan de la Cierva Program. M.D.B. acknowledges the Generalitat de Catalunya for the ICREA Acadèmia Program.

\section{REFERENCES}

Arevalo-Lopez, A.M. \& Alario-Franco, M.A. (2009). Reliable method for determining the oxidation state in chromium oxides. Inorg Chem 48, 11843-11846.

Botton, G.A., Appel, C.C., Horsewell, A. \& StobBs, W.M. (1995). Quantification of the EELS near-edge structures to study Mn doping in oxides. J Microsc-Oxf 180, 211-216.

Cave, L., Al, T., Loomer, D., Cogswell, S. \& Weaver, L. (2006). A STEM/EELS method for mapping iron valence ratios in oxide minerals. Micron 37, 301-309.

Colliex, C., Manoubi, T. \& Ortiz, C. (1991). Electron-energy-lossspectroscopy near-edge fine-structures in the iron-oxygen system. Phys Rev B 44, 11402-11411.

Daulton, T.L., Little, B.J., Lowe, K. \& Jones-Meehan, J. (2002). Electron energy loss spectroscopy techniques for the study of microbial chromium (VI) reduction. J Microbiol Meth 50, 39-54.

EgerTon, R.F. (1996). Electron Energy-Loss Spectroscopy in the Electron Microscope. New York, NY, USA: Plenum Press.

Estrader, M., López-Ortega, A., Estradé, S., Golosovsky, I.V., Salazar-Alvarez, G., Vasilakaki, M., Trohidou, K.N. Varela, M., Stanley, D.C., Sinko, M., Pechan, M.J., Keavney, D.J., Peiró, F., Suriñach, S., Baró, M.D. \& Nogú́s, J. (2013). Robust antiferromagnetic coupling in hard-soft bi-magnetic core/shell nanoparticles. Nat Commun 4, 2960.

Garvie, L. \& Craven, A. (1994). High-resolution parallel electronenergy-loss spectroscopy of $\mathrm{Mn} \mathrm{L}(2,3)$-edges in inorganic manganese compounds. Phys Chem Miner 21, 191-206. 
Garvie, L.A.J. \& Buseck, P.R. (1998). Ratios of ferrous to ferric iron from nanometre-sized areas in minerals. Nature 396, 667-670.

Gilbert, B., Frazer, B., Belz, A., Conrad, P., Nealson, K., Haskel, D., Lang, J., Srajer, G. \& De Stasio, G. (2003). Multiple scattering calculations of bonding and X-ray absorption spectroscopy of manganese oxides. J Phys Chem A 107, 2839-2847.

Gloter, A., Serin, V., Turquat, C., Cesari, C., Leroux, C. \& Niнoul, G. (2001). Vanadium valency and hybridization in $\mathrm{V}$-doped hafnia investigated by electron energy loss spectroscopy. Eur Phys J B 22, 179-186.

Graetz, J., Ahn, C., Ouyang, H., Rez, P. \& Fultz, B. (2004). White lines and $\mathrm{d}$-band occupancy for the $3 \mathrm{~d}$ transition-metal oxides and lithium transition-metal oxides. Phys Rev B 69, 235103.

Gubbens, A., Barfels, M., Trevor, C., Twesten, R., Mooney, P., Thomas, P., Menon, N., Kraus, B., Mao, C. \& McGinn, B. (2010). The GIF quantum, a next generation post-column imaging energy filter. Ultramicroscopy 110, 962-970.

Kourkoutis, L.F., HotTa, Y., Susaki, T., Hwang, H.Y. \& Muller, D.A. (2006). Nanometer scale electronic reconstruction at the interface between $\mathrm{LaVO}_{3}$ and $\mathrm{LaVO}_{4}$. Phys Rev Lett 97, 256803.

Kurata, H. \& Colliex, C. (1993). Electron-energy-loss core-edge structures in manganese oxides. Phys Rev B 48, 2102-2108.

LAfFont, L. \& Gibot, P. (2010). High resolution electron energy loss spectroscopy of manganese oxides: Application to $\mathrm{Mn}_{3} \mathrm{O}_{4}$ nanoparticles. Mater Charact 61, 1268-1273.

Leapman, R.D., Grunes, L.A. \& Fejes, P.L. (1982). Study of the $\mathrm{L}_{2,3}$ edges in the $3 \mathrm{~d}$ transition metals and their oxides by electronenergy-loss spectroscopy with comparisons to theory. Phys Rev B 26, 614-635.

Logvenov, G., Gozar, A. \& Bozovic, I. (2009). High-temperature superconductivity in a single copper-oxygen plane. Science 326, 699-702.

Loomer, D.B., Al, T.A., Weaver, L. \& Cogswell, S. (2007). Manganese valence imaging in Mn minerals at the nanoscale using STEM-EELS. Am Mineral 92, 72-79.

Lopez-Ortega, A., Estrader, M., Salazar-Alvarez, G., Estrade, S., Golosovsky, I.V., Dumas, R.K., Keavney, D.J., Vasilakaki, M., Trohidou, K.N., Sort, J., Peiro, F., Surinach, S., Baro, M.D. \& Nogues, J. (2012). Strongly exchange coupled inverse ferrimagnetic soft/hard, $\mathrm{Mn}_{\mathrm{x}} \mathrm{Fe}_{3-\mathrm{x}} \mathrm{O}_{4} / \mathrm{Fe}_{\mathrm{x}} \mathrm{Mn}_{3-\mathrm{x}} \mathrm{O}_{4}$, core/shell heterostructured nanoparticles. Nanoscale 4, 5138-5147.

Luo, W., Franceschetti, A., Varela, M., Tao, J., Pennycook, S.J. \& Pantelides, S.T. (2007). Orbital-occupancy versus charge ordering and the strength of electron correlations in electrondoped $\mathrm{CaMnO}_{3}$. Phys Rev Lett 99, 036402.

Luo, W., Varela, M., Tao, J., Pennycook, S.J. \& Pantelides, S.T. (2009). Electronic and crystal-field effects in the fine structure of electron energy-loss spectra of manganites. Phys Rev B 79, 052405.

McNaught, A.D. \& Wilkinson, A. (1997). IUPAC. Compendium of Chemical Terminology (the "Gold Book"). Oxford, UK: Blackwell Scientific Publications.

Meneses, C.T., Vicentin, F.C., SASAKi, J.M. \& Macedo, M.A. (2007). Influence of $\mathrm{Li}$ on the $\mathrm{K}$-edge of $\mathrm{O}$ and $\mathrm{L}_{2,3}$ of the Mn XANES in $\mathrm{Li}_{\mathrm{x}} \mathrm{Mn}_{2} \mathrm{O}_{4}$ thin films. J Electron Spectrosc Rel Phen 156, 326-328.

Paterson, J.H. \& KrivaneK, O.L. (1990). ELNES of 3d transitionmetal oxides: II. Variations with oxidation state and crystal structure. Ultramicroscopy 32, 319-325.
Pearson, D.H., Ahn, C.C. \& Fultz, B. (1993). White lines and d-electron occupancies for the $3 \mathrm{~d}$ and $4 \mathrm{~d}$ transition metals. Phys $\operatorname{Rev} B$ 47, 8471.

Rask, J., Miner, B. \& Buseck, P. (1987). Determination of manganese oxidation-states in solids by electron energy-loss spectroscopy. Ultramicroscopy 21, 321-326.

Riedl, T., Gemming, T., Gruner, W., Acker, J. \& Wetzig, K. (2007). Determination of manganese valency in $\mathrm{La}_{1-\mathrm{x}} \mathrm{Sr}_{\mathrm{x}} \mathrm{MnO}_{3}$ using ELNES in the (S)TEM. Micron 38, 224-230.

Riedl, T., Gemming, T. \& Wetzig, K. (2006). Extraction of EELS white-line intensities of manganese compounds: Methods, accuracy, and valence sensitivity. Ultramicroscopy 106, 284-291.

Riedl, T., Serra, R., Calmels, L. \& Serin, V. (2008). Valence sensitivity of $\mathrm{Fe}-\mathrm{L}_{2,3}$ white-line ratios extracted from EELS. In EMC 2008, Volume 1: Instrumentation and Methods, M. Luysberg, K. Tillmann \& Thomas Weirich (Eds.), pp. 419-420). Berlin, Germany: Springer-Verlag Berlin Heidelberg.

Salazar-Alvarez, G., Lidbaum, H., Lopez-Ortega, A., Estrader, M., Leifer, K., Sort, J., Surinach, S., Baro, M.D. \& Nogues, J. (2011). Two-, three-, and four-component magnetic multilayer onion nanoparticles based on iron oxides and manganese oxides. J Am Chem Soc 133, 16738-16741.

SCHMid, H.K. \& MADER, W. (2006). Oxidation states of Mn and Fe in various compound oxide systems. Micron 37, 426-432.

Sherman, D.M. (1984). The electronic-structures of manganese oxide minerals. Am Mineral 69, 788-799.

Suchorski, Y., Rihko-Struckmann, L., Klose, F., Ye, Y., Alandjiyska, M., Sundmacher, K. \& Weiss, H. (2005). Evolution of oxidation states in vanadium-based catalysts under conventional XPS conditions. Appl Surf Sci 249, 231-237.

Sun, J., Krusinelbaum, L., Duncombe, P., Gupta, A. \& Laibowitz, R. (1997). Temperature dependent, non-ohmic magnetoresistance in doped perovskite manganate trilayer junctions. Appl Phys Lett 70, 1769-1771.

TAFTO, J. \& KRIVANEK, O.L. (1982). Site-specific valence determination by electron energy-loss spectroscopy. Phys Rev Lett 48, 560-563.

Tan, H., Verbeeck, J., Abakumov, A. \& Van Tendeloo, G. (2012). Oxidation state and chemical shift investigation in transition metal oxides by EELS. Ultramicroscopy 116, 24-33.

Turquat, C., Leroux, C., Gloter, A., Serin, V. \& Nihoul, G. (2001). $\mathrm{V}$-doped $\mathrm{HfO}_{2}$ : Thermal stability and vanadium valence. Int $\mathrm{J}$ Inorg Mater 3, 1025-1032.

VAn Aken, P.A., Liebscher, B. \& Styrsa, V.J. (1998). Quantitative determination of iron oxidation states in minerals using Fe L2,3 -edge electron energy-loss near-edge structure spectroscopy. Phys Chem Miner 25, 323-327.

Varela, M., Oxley, M.P., Luo, W., TaO, J., Watanabe, M., Lupini, A.R., Pantelides, S.T. \& Pennycook, S.J. (2009). Atomic-resolution imaging of oxidation states in manganites. Phys Rev B 79, 085117.

Wang, Y.Q., Maclaren, I. \& Duan, X.F. (2001). EELS analysis of manganese valence states in rare-earth manganites $\left(\mathrm{La}_{1-\mathrm{x}} \mathrm{Y}_{\mathrm{x}}\right)_{0.5}\left(\mathrm{Ca}_{1-\mathrm{y}} \mathrm{Sr}_{\mathrm{y}}\right)_{0.5} \mathrm{MnO}_{3}$. Mat Sci Eng A 318, 259-263.

Zhang, S., Livi, K.J.T., Gaillot, A., Stone, A.T. \& Veblen, D.R. (2010). Determination of manganese valence states in $\left(\mathrm{Mn}^{3+}\right.$, $\mathrm{Mn}^{4+}$ ) minerals by electron energy-loss spectroscopy. Am Mineral 95, 1741-1746. 